\title{
Single v. multiple measures of skin carotenoids by resonance Raman spectroscopy as a biomarker of usual carotenoid status
}

\author{
Stephanie Scarmo ${ }^{1}$, Brenda Cartmel ${ }^{1,2}$, Haiqun Lin $^{1}$, David J. Leffell ${ }^{2,3}$, Igor V. Ermakov ${ }^{4}$, \\ Werner Gellermann ${ }^{4}$, Paul S. Bernstein ${ }^{5}$ and Susan T. Mayne ${ }^{1,2 *}$ \\ ${ }^{1}$ Department of Chronic Disease Epidemiology, Yale School of Public Health, 60 College Street, PO Box 208034, \\ New Haven, CT 06520-8034, USA \\ ${ }^{2}$ Yale Cancer Center, New Haven, CT, 06520, USA \\ ${ }^{3}$ Department of Dermatology and Surgery, Yale University School of Medicine, New Haven, CT O6510, USA \\ ${ }^{4}$ Department of Physics and Astronomy, University of Utah, Salt Lake City, UT 84122, USA \\ ${ }^{5}$ Department of Ophthalmology and Visual Sciences, Moran Eye Center, University of Utah School of Medicine, \\ Salt Lake City, UT 84132, USA \\ (Submitted 14 June 2012 - Final revision received 4 December 2012 - Accepted 5 December 2012 - First published online 28 January 2013)
}

\begin{abstract}
Resonance Raman spectroscopy (RRS) is a non-invasive method of assessing carotenoid status in the skin, which has been suggested as an objective indicator of fruit/vegetable intake. The present study assessed agreement and identified predictors of single $v$. multiple RRS measures of skin carotenoid status. A total of seventy-four participants had their skin carotenoid status measured in the palm of the hand by RRS at six time points over 6 months. Questionnaires were administered to collect information on demographic, lifestyle and dietary data. Mean age of the participants was $36 \cdot 6$ years, $62 \cdot 2 \%$ were female, $83 \cdot 8 \%$ Caucasian and $85 \cdot 1 \%$ were non-smoking at baseline. There was a good agreement between a single measure of skin carotenoids by RRS and multiple measures (weighted $\kappa=0 \cdot 80 ; 95 \%$ CI $0 \cdot 72,0 \cdot 88)$. The same variables were significantly associated with carotenoid status based on single or multiple measures, including a positive association with intake of total carotenoids $(P<0 \cdot 01)$ and an inverse association with season of measurement $(P \leq 0 \cdot 05)$. The exception was recent sun exposure, which emerged as a significant predictor of lower carotenoid status only when using multiple RRS measures $(P \leq 0 \cdot 01)$. A single RRS measure was reasonably accurate at classifying usual skin carotenoid status. Researchers using RRS may want to take into account other factors that are associated with the biomarker, including season of measurement and recent sun exposure.
\end{abstract}

\section{Key words: Biomarkers: Resonance Raman spectroscopy: Carotenoids: Fruits/vegetables}

Carotenoids are commonly ingested plant pigments found in fruits and vegetables that are responsible for red, orange and yellow colouring. Evidence suggests that a high carotenoid intake is associated with lower incidence of several chronic diseases $^{(1-3)}$. Because carotenoids are not found in significant concentrations in other foods, but are widely distributed in fruits and vegetables, they are considered to be the best current biomarker for fruit and vegetable intake ${ }^{(4)}$, and levels of carotenoids in blood and tissue are known to correlate with dietary carotenoid intake ${ }^{(5)}$. Methods for assessing carotenoid intake typically involve self-report from dietary questionnaires, which can be subject to bias and measurement error ${ }^{(6)}$, or biochemical analysis (HPLC) of plasma or tissue samples to estimate carotenoid status objectively $^{(7,8)}$, which is invasive and expensive.

Growing evidence supports the validity of resonance Raman spectroscopy (RRS) as an alternative to HPLC for the measurement of carotenoids in living tissues, and studies around the world are now using this technology in research settings ${ }^{(9-14)}$. A novel, non-invasive technique for measuring carotenoid status in the skin using visible light, RRS utilises a small probe with a laser at a blue wavelength $(\lambda=488 \mathrm{~nm})$ to objectively measure total carotenoid levels in skin ${ }^{(15)}$. Our group has previously shown that RRS is a reliable (reproducible) and valid method for estimating skin carotenoid status, when compared with HPLC analyses of biopsied skin in healthy adults, with correlation coefficients ranging from $r 0.7^{(9)}$ to $0 \cdot 9^{(10)}$. We $\mathrm{We}^{(12)}$ and others ${ }^{(16)}$ have been evaluating skin carotenoid status as a biomarker of carotenoid or fruit and vegetable intake in cross-sectional studies, as well as in studies of carotenoid or fruit and vegetable interventions ${ }^{(17-20)}$.

With the exception of reference biomarkers of nutritional status (e.g. doubly-labelled water and urinary nitrogen),

Abbreviation: RRS, resonance Raman spectroscopy.

*Corresponding author: Dr S. T. Mayne, fax +1 203785 6980, email susan.mayne@yale.edu 
most other biomarkers of nutritional status can be affected by factors other than dietary intake. For example, plasma 25-hydroxyvitamin $\mathrm{D}$ is used as a biomarker of vitamin $\mathrm{D}$ status, but circulating levels are known to vary seasonally with UV exposure ${ }^{(21-23)}$ and also to be affected by factors such as obesity ${ }^{(24)}$ and skin pigmentation ${ }^{(22,25)}$, which researchers often take into account in studies using this biomarker. Given the interest in using RRS to measure skin carotenoid status as a biomarker, studies examining possible factors that may have an impact on the nutrient biomarker are needed. In our prior publication validating RRS measures against skin biopsies, we reported how mean baseline RRS values varied as a function of selected variables of interest (univariate analysis only) ${ }^{(9)}$. Because some of those selected variables are associated with each other, the purpose of the present analysis was two-fold: (a) to extend our prior work by performing multivariate analyses to identify significant, independent predictors of carotenoid status and (b) to examine how one RRS measure performed as a marker of status, as compared with multiple measures over time. Identification of factors (beyond carotenoid intake) that correlate with 'usual' carotenoid status allows investigators, when appropriate, to take into account those factors when interpreting data that use RRS as a biomarker of diet and or/nutrient status.

\section{Subjects and methods}

\section{Study population}

The Skin Antioxidant Assessment Study was a prospective investigation to examine the reliability and validity of RRS as a measure of skin carotenoid status in adults ${ }^{(9)}$. A total of seventy-five subjects participated in the study, which assessed the intra- and interperson variability in carotenoid status, measured by RRS within a body site (palm, inner forearm and outer forearm), across these different body sites and over time. Details of the study recruitment are reported elsewhere $^{(9)}$. Briefly, participants were healthy adults, aged 21 to 65 years, who resided in the state of Connecticut, in the northeastern USA. Since smokers are known to have lower plasma carotenoid concentrations than non-smokers ${ }^{(26,27)}$, we recruited both smokers and non-smokers in our sample. The present study was conducted according to the guidelines laid down in the Declaration of Helsinki, and all procedures involving human subjects were approved by the Institutional Review Board at the Yale University School of Medicine. Written informed consent was obtained from all subjects.

\section{Data collection}

Subject characteristics. Participation in the study required subjects to complete six visits at the following time points: baseline, 1 week, 2 weeks, 1, 3 and 6 months. The 6-month follow-up period allowed us to capture maximal seasonal variation in dietary intake of fruits/vegetables. At the baseline visit, a questionnaire was administered in-person by a trained interviewer to collect information on basic demographics (sex, age, marital status, education level, usual height and weight, use of tobacco and alcohol, diet, supplement use and self-identified race/ethnicity). Participants were also asked to self-identify skin colour (inner arm), under the guidance of a research assistant, using a colour wheel of skin tone samples provided by a prosthetics manufacturer (Steeper USA(C). At the baseline and at each follow-up visit, the season of RRS measurement was recorded and the individuals were asked to estimate the number of cigarettes smoked per week (later collapsed into current smoking status; yes $v$. no) and the number of hours during the past $3 \mathrm{~d}$ their skin had been exposed to the sun without sunscreen.

Resonance Raman spectroscopy. Details of RRS instrumentation have been reported elsewhere ${ }^{(10-12,28)}$. To assess the reproducibility of the instrument, RRS scans were performed at baseline and at follow-up visits on all participants to assess skin carotenoid status in the palm, inner arm and outer arm over a period of 6 months. On each day when the measurements were taken, the instrument was calibrated with an external diamond standard, and laser power was measured to assess the potential drift. A single body site was scanned three times for reliability, and the mean value of the Raman measure from a body site was used in data analysis. Mean calibration-adjusted values were used in this analysis.

Dietary intake data. All participants were required to complete a self-administered FFQ from the Nutrition Assessment Shared Resource at the Fred Hutchinson Cancer Research Center (Seattle, Washington, USA) during the baseline and at the 6-month follow-up visit. This ten-page diet questionnaire has been validated in the Women's Health Initiative Dietary Assessment Study for nutrient content from foods (Pearson $r 0.52$ for $\beta$-carotene estimated from the FFQ and mean of $8 \mathrm{~d}$ of intake from $24 \mathrm{~h}$ recalls) ${ }^{(29)}$. Information was collected on the amount and frequency of usual food and drink consumption over the past 2 months, including questions on seasonal consumption of selected fruits and vegetables. Responses provided an estimate of daily food servings consumed, as well as nutrients associated with those servings for each study participant, including intake of total dietary carotenoids. Participants currently taking nutrient supplements at the baseline visit, such as multivitamins that may contain carotenoids, were asked to bring in bottles and continue taking the supplement for the 6-month study period. If participants were not currently consuming a nutrient supplement at baseline, they were asked to refrain from supplements until the study had concluded. No subjects reported taking single-nutrient carotenoid supplements, so the only source of supplemental carotenoids was multivitamins in the present study. The number of pills consumed per week and the nutrient content of $\beta$-carotene, lycopene and lutein in the multivitamins was recorded. Intake of total carotenoids ( $\mathrm{mg} / \mathrm{d}$ ) was estimated by summing carotenoids consumed from food and supplements.

\section{Statistical analysis}

Mean RRS scores over time (average of six time points), indicative of 'usual' carotenoid status, were examined by demographic characteristics. A repeated-measures ANOVA was used to test whether mean RRS scores were significantly 
different by subject characteristics. To avoid having a stratum with one observation, the subject with race marked 'other' was coded as missing for this variable.

Main predictors of skin carotenoid status were examined in adjusted analyses at baseline by multivariate linear regression and over time (skin carotenoid status measured by RRS at six time points over 6 months) by linear mixed effects regression. The linear mixed effects models were constructed with time as a six-level class variable, random intercept and a first-order autoregressive $^{(1)}$ covariance structure for the within-subject errors. The longitudinal models took into account the timing of the RRS measurement ( $v$. an average) and accounted for any other time-dependent variables (e.g. season of RRS measurement, recent sun exposure and current smoking status) as well as time-fixed variables assessed at baseline only (e.g. race/ethnicity, skin tone, BMI and usual diet). Because we were interested in the association of usual diet with RRS (rather than how RRS changed with diet in this non-intervention study), we used the calculated average intake of total carotenoids from the baseline and 6-month diet assessment in the models. There is evidence suggesting that energy adjustment provides a more reliable estimate of dietary intake ${ }^{(30)}$. As a result, we adjusted total carotenoid intake for total energy consumed per $\mathrm{d}$. The final multivariate models were based on backwards selection, with some forced predictors (e.g. BMI and current smoking status) selected a priori, based on preliminary data from our previous analysis $^{(9)}$, as well as other literature indicating that both BMI and current smoking status correlate with plasma carotenoids ${ }^{(26,27,31)}$

A cross-tabulation was constructed to compare the agreement between subjects' baseline and usual skin carotenoid status (here, mean skin carotenoid status measured at six time points), examined by quartiles. A simple and weighted $\kappa$ statistic was estimated to assess agreement. Because the six measures were not taken in equal time increments, we also examined agreement only using the average of the baseline, 3- and 6-month time points as the estimate of 'usual'. All statistical computations were conducted using SAS version 9.2 (SAS Institute, Inc.).

\section{Results}

A full description of the study population has been reported previously $^{(9)}$. Of the seventy-five subjects recruited for the present study, one participant came in for the baseline scan but did not complete the baseline questionnaire or any subsequent scanning visits, and therefore was excluded from all analyses. A total of seventy-four people were included in the final study sample, with a mean age of 36.6 years (median 33 years) and $62.2 \%$ female. The majority of the population was identified as Caucasian (83.8\%) and nonsmoking at baseline $(85 \cdot 1 \%)$. We observed the lowest usual skin carotenoid status (here, the average of six measures without accounting for time) among African American subjects, while Asian subjects had a significantly higher usual status $(P<0 \cdot 01)$ compared with Caucasians. Baseline smoking status $(P=0.07)$ and dark skin tone $(P=0.06)$ were both marginally significant predictors of lower usual skin carotenoid status in univariate analyses.

We examined the main predictors of skin carotenoid status at baseline (one measure only) and over time (longitudinally, using all six independent measurements to account for time) in multivariate analysis (Table 1). We observed that many of

Table 1. Main predictors of skin carotenoid status at baseline and over time*, with adjustment for covariates

(Mean values and standard deviations; number of subjects and percentages)

\begin{tabular}{|c|c|c|c|c|c|c|c|c|}
\hline \multirow[b]{2}{*}{ Subject characteristics } & \multicolumn{4}{|c|}{ At baseline } & \multicolumn{2}{|c|}{ Baseline } & \multicolumn{2}{|c|}{ Over 6 months } \\
\hline & $n$ & $\%$ & Mean & SD & $\beta$ & $P$ & $\beta$ & $P$ \\
\hline Intake of total carotenoids $(\mathrm{mg} / \mathrm{d}) \dagger$ & & & $17 \cdot 0$ & 8.9 & $0.28 \ddagger$ & $<0.01$ & $0.23 \ddagger$ & $<0.01$ \\
\hline Recent sun exposure $(\mathrm{h} / \mathrm{d}) \ddagger$ & & & 1.0 & 1.5 & $0.004 \ddagger$ & 0.96 & $-0.06 \ddagger$ & 0.01 \\
\hline \multicolumn{9}{|l|}{ Skin tone } \\
\hline Light & 62 & 83.8 & & & Reference & $\mathrm{N} / \mathrm{A}$ & Reference & N/A \\
\hline Medium & 9 & $12 \cdot 2$ & & & -0.14 & 0.44 & -0.14 & 0.38 \\
\hline Dark & 3 & $4 \cdot 0$ & & & -0.52 & 0.19 & -0.57 & 0.12 \\
\hline \multicolumn{9}{|l|}{ Season of RRS§ measurement } \\
\hline Winter & 10 & 13.5 & & & Reference & N/A & Reference & N/A \\
\hline Spring/autumn & 49 & $66 \cdot 2$ & & & -0.20 & $0 \cdot 18$ & $-0 \cdot 10$ & 0.01 \\
\hline Summer & 15 & $20 \cdot 3$ & & & -0.38 & 0.05 & -0.22 & $<0.01$ \\
\hline \multicolumn{9}{|l|}{ Race } \\
\hline White & 62 & 83.8 & & & Reference & N/A & Reference & N/A \\
\hline Asian & 6 & $8 \cdot 1$ & & & 0.82 & $<0.01$ & 0.54 & $<0.01$ \\
\hline African American & 5 & $6 \cdot 8$ & & & -0.15 & 0.65 & -0.07 & 0.80 \\
\hline Other & 1 & 1.3 & & & Not estimated & & Not estimated & \\
\hline \multicolumn{9}{|l|}{ Current smoker } \\
\hline No & 63 & $85 \cdot 1$ & & & Reference & N/A & Reference & N/A \\
\hline Yes & 11 & 14.9 & & & -0.29 & 0.07 & -0.10 & 0.17 \\
\hline BMI $\left(\mathrm{kg} / \mathrm{m}^{2}\right)$ & & & $24 \cdot 2$ & $4 \cdot 0$ & $0.01 \ddagger$ & 0.93 & $-0.04 \ddagger$ & 0.50 \\
\hline
\end{tabular}

N/A, not applicable; RRS, resonance Raman spectroscopy.

* Skin carotenoid status measured by RRS at six time points over 6-months (baseline, 1-week, 2-week, 1-month, 3-month and 6-month follow-up visits).

† Including carotenoid intake from supplements; adjusted for mean daily energy intake (average of baseline and 6-month estimates).

$\ddagger$ To make $\beta$-coefficients more interpretable, they are scaled to the interquartile range for each variable as follows (total carotenoids per $10 \mathrm{mg} / \mathrm{d}$, recent sun exposure per $2 \mathrm{~h} / \mathrm{d}$ and BMI per $5 \mathrm{~kg} / \mathrm{m}^{2}$ ).

$\S$ Mean RRS values for total study population at baseline was 1.55 (SD 0.57 ) counts. 
Table 2. Agreement between baseline and usual ${ }^{\star}$ skin carotenoid status quartiles, measured by resonance Raman spectroscopy at six time points $(n 74) \dagger$

\begin{tabular}{lrrrrr}
\hline & \multicolumn{4}{c}{ Usual carotenoid status* } \\
\cline { 2 - 5 } Baseline carotenoid status & Q4 & Q3 & Q2 & Q1 & Total \\
\hline Q4 & 14 & 4 & 0 & 0 & 18 \\
Q3 & 4 & 12 & 3 & 0 & 19 \\
Q2 & 0 & 3 & 14 & 2 & 19 \\
Q1 & 0 & 0 & 2 & 16 & 18 \\
Total & 18 & 19 & 19 & 18 & 74
\end{tabular}

$Q$, quartile.

* Mean skin carotenoid status in the palm measured at six time points; Q4 is highest quartile (baseline, 1-week, 2-week, 1-month, 3-month and 6-month follow-up visits).

† Simple $\kappa=0.68(95 \% \mathrm{Cl} 0.54,0.81)$; weighted $\kappa=0.80(95 \% \mathrm{Cl} 0.72,0.88)$.

the same factors were independent predictors of skin carotenoid status both at baseline and over time, with adjustment for covariates. After taking into account dietary intake of total carotenoids, which as expected was significantly predictive of higher skin carotenoid status at baseline and over time $(P<0.01$ for both), we observed that season of measurement and race were also independently predictive of skin carotenoid status, measured by RRS. In particular, significantly lower skin carotenoid status was observed when RRS measurements were obtained during the summer months ( $P=0.05$ at baseline; $P<0.01$ over time), followed by spring/autumn measurements (not significant at baseline; $P<0.01$ over time), as compared with the winter season. Asian race was significantly associated with higher skin carotenoid status at baseline and over time $(P<0.01$ for both), while African American race was associated with a non-significant lower status; Caucasian race was the comparison group. Current smoking status was marginally associated with lower carotenoid status at baseline $(P=0 \cdot 07)$. Finally, self-reported recent sun exposure (number of hours in the $3 \mathrm{~d}$ prior to RRS measurement when skin had been exposed to the sun without sunscreen) emerged as a significant predictor of skin carotenoid status over time $(P=0 \cdot 01)$, while it was not a significant predictor when using only one measure of sun exposure taken at the baseline visit $(P=0 \cdot 96)$

We observed good agreement between skin carotenoid status measured by RRS at baseline and multiple time points. Of the eighteen subjects categorised in the highest quartile at baseline, fourteen remained in the highest group when carotenoid status was estimated as the average of six time points. In all, sixteen of the eighteen subjects categorised as having the lowest carotenoid status at baseline remained in the lowest category when their status was averaged over time. Overall, fifty-six of the total seventy-four subjects remained in the same category of skin carotenoid status, and the weighted $\kappa$ showed an $80 \%$ agreement (95\% CI $0 \cdot 72$, 0.88 ) between the measures (Table 2). When we repeated the analysis, restricting the data to baseline, 3- and 6-month follow-up visits (so the usual measure of carotenoid status was less influenced by the earlier measures), the weighted $\kappa$ showed a $69 \%$ agreement $(95 \%$ CI $0.59,0.80)$ between the measures (Table 3).

\section{Discussion}

We previously reported on the predictors of skin carotenoid status in univariate analysis at baseline only ${ }^{(9)}$. Because many of those covariates were correlated, here, we report the results of a multivariate analysis to identify predictors of carotenoid status, with adjustment for other factors that correlate with the RRS biomarker. As expected, many of the predictors identified for usual carotenoid status (e.g. over time) were the same as those identified at one time point, including self-reported intake of total carotenoids and summer season of RRS measurement.

In the present analyses, we used an average of the baseline and 6-month dietary intake, which minimises the ability to detect changes in an individual's diet over time but allows us to capture usual diet. Evidence suggests that there are seasonal effects on fruit and vegetable consumption, with variation in the availability and variety of these foods ${ }^{(32,33)}$, which is probably reflected in overall carotenoid status in blood and tissue. The half-life of carotenoids in skin is believed to be longer than the half-life in blood (the latter being approximately 2 weeks) ${ }^{(34)}$, and our own intervention data suggest that skin reflects carotenoid intake over the past $8+$ weeks (with values still increasing at 8 weeks after feeding subjects a high-carotenoid diet ${ }^{(20)}$. Therefore, the lower RRS values that we observed during the summer months (June, July and August) may, in part, reflect dietary intake from the late winter/early spring months (March, April and May), when the availability and variety of fruits and vegetables is more limited in the northeast USA.

In multivariate analysis, self-reported sun exposure (queried at all six time points) emerged as a significant predictor of skin carotenoid status over time, and one that was not significant using baseline-only sun exposure (one time point) in relation to baseline RRS measures. Few studies have examined the effect of UV radiation on carotenoid concentrations in human skin, and most assessed $\beta$-carotene only with a variety of measurement techniques, making it difficult to directly compare study results ${ }^{(35)}$. Experimental studies suggest that skin carotene and retinol concentrations decrease immediately following UV radiation in hairless rodents, but levels can be recovered with carotenoid supplementation $^{(36)}$. There is also evidence from human studies to suggest that the degradation of skin caroten-

Table 3. Agreement between baseline and usual* skin carotenoid status quartiles, measured by resonance Raman spectroscopy at three time points $(n 74) \dagger$

\begin{tabular}{lrrrrr}
\hline & \multicolumn{4}{c}{ Usual carotenoid status* } \\
\cline { 2 - 4 } Baseline carotenoid status & Q4 & Q3 & Q2 & Q1 & Total \\
\hline Q4 & 13 & 5 & 0 & 0 & 18 \\
Q3 & 5 & 8 & 6 & 0 & 19 \\
Q2 & 0 & 6 & 10 & 3 & 19 \\
Q1 & 0 & 0 & 3 & 15 & 18 \\
Total & 18 & 19 & 19 & 18 & 74
\end{tabular}

Q, quartile.

* Mean skin carotenoid status in the palm measured at three time points (baseline, 3-month and 6-month follow-up visits); Q4 is highest quartile.

† Simple $\kappa=0.50(95 \% \mathrm{Cl} 0.35,0.64)$; weighted $\kappa=0.69(95 \% \mathrm{Cl} 0.59,0.80)$. 
oids following exposure to UV radiation may be differential, with more lycopene being degraded than $\beta$-carotene ${ }^{(37)}$, possibly serving as the first line of defence in human skin photoprotection, with lycopene being a predominant carotenoid in human $\operatorname{skin}^{(38)}$. Because the present findings suggest that recent UV radiation from sun exposure is correlated with lower carotenoid status at a localised body site, it may be helpful to consider adjusting for sun exposure in future studies using RRS as a biomarker. It is important to highlight that our study subjects were queried on recent sun exposure (only reporting on the $3 \mathrm{~d}$ prior to RRS scans); we observed a range of 3-d sun exposure measured at baseline from zero to eighteen total hours, with the average 3 -d sun exposure equalling to $3 \mathrm{~h}$. While recent sun exposure was significantly inversely correlated with RRS, the magnitude of the effect ( $\beta$ coefficient) was modest, suggesting a relatively minor overall effect of UV radiation on RRS measures, at least under the conditions of sun exposure that we observed. Because we had no measure of overall sun exposure over the duration of 6 months, our multivariate models could only control for recent exposure, which may or may not be correlated with an individual's usual sun exposure. Thus, the lower levels of skin carotenoids that we observed when RRS measurements were taken in summer could also, in part, be due to greater sun exposure. Future studies of RRS in subjects with extensive sun exposure are needed to better understand the impact of sun exposure on the RRS biomarker of skin carotenoid status.

A significant association was observed between selfidentified race and skin carotenoid status. Asian subjects had significantly higher skin carotenoid status, compared with white and African American subjects; however, our sample included only six Asian subjects. This may be a chance finding given the small sample size, but it is plausible that the higher status in Asians reflects their lifestyle patterns or genetic differences. While genetics might also explain some of the low skin carotenoid status in our African American subjects, another possibility is greater melanin content or differences in body composition (adiposity). With our limited sample size, consisting of only three individuals with dark skin tone and only five individuals who identified as African American, we were unable to examine this further with multivariate modelling. However, as previously reported ${ }^{(9)}$, we examined the ratio of measured RRS values in the outer arm (area of most skin pigmentation) $v$. the palm (area of least skin pigmentation) among participants who were identified as dark skinned, as compared with similar ratios obtained from subjects with light skin. We observed no evidence that the RRS ratios varied significantly with skin pigmentation, as might be expected if melanin had a filtering effect on the laser ${ }^{(9)}$. Nevertheless, we measured the palm of the hand in our studies, with one of the reasons being that the dermal melanin is light and less variable among subjects of different races and ethnicities. Additional research in racially diverse populations with larger sample sizes and objective quantitation of skin pigmentation is needed to explain the true effect, if any, of melanin on the RRS measures.

The present results indicate that a single RRS measure of the palm of the hand was a reasonably good indicator of a subjects' usual skin carotenoid status. Multiple measures did not greatly improve the ability of the biomarker to objectively categorise carotenoid status. In terms of feasibility of use, a single RRS measure can save follow-up efforts in studies of the biomarker and health outcomes. Among those participants for whom one measure of skin carotenoid status misclassified the usual skin carotenoid status (by quartiles), the degree of misclassification did not exceed one quartile in difference. This suggests that a single RRS measure can be used in future studies to identify subjects with low carotenoid status, who may be at risk for subsequent nutrition-related diseases.

A strength of the present study was the prospective design, which provided up to six repeated measurements for each participant over the course of a 6-month follow-up in conditions with marked seasonal variation in climate and availability of fresh fruit and vegetables. A limitation of the present study was the use of self-reported data for estimating sun exposure, as well as the lack of an objective measure of melanin. The primary limitation was the small sample size, consisting primarily of Caucasian non-smoking women.

In summary, results from the present study suggest that a single measure of skin carotenoids by RRS is a reasonably robust indicator of usual carotenoid status. We also identified factors, such as season of measurement and sun exposure, which correlate with skin carotenoid status. These findings contribute to a growing body of research advancing the development of this innovative nutrition-related biomarker.

\section{Acknowledgements}

We wish to thank the participants in the present study. This study was supported by grants R01 CA096838 (S. T. M.), sub-award no. A05202 and M03A00158 (I. V. E. and W. G.) and R01 EY11600 (P. S. B.) from the National Institutes of Health, USA; and an unrestricted departmental grant from the Research to Prevent Blindness (New York, NY, USA) (P. S. B.). All authors have made substantial contributions and the final approval of the conceptions, drafting and the final version of this manuscript. The authors' contributions are as follows: S. S. was involved in the recruitment of the study subjects and data collection, statistical analysis and manuscript drafting and review; B. C. contributed to the experimental design, supervision of the human study, data collection and manuscript review; H. L. contributed to the experimental design, advised on the statistical analysis and manuscript review; D. J. L. contributed to the experimental design, clinical support and manuscript review; I. V. E. provided spectroscopy support and assisted with training and data interpretation, and manuscript review; P. S. B. was involved in the experimental design and manuscript review; W. G. participated in the design and construction of the RRS system, oversaw training for the data collection and manuscript review; S. T. M. contributed to the experimental design, acquiring funding and manuscript review. W. G. and P. S. B. hold patents for the methods described in the present paper. The authors report no other competing interests. 


\section{References}

1. Michaud DS, Feskanich D, Rimm EB, et al. (2000) Intake of specific carotenoids and risk of lung cancer in 2 prospective US cohorts. Am J Clin Nutr 72, 990-997.

2. Krinsky NI \& Johnson EJ (2005) Carotenoid actions and their relation to health and disease. Mol Aspects Med 26, 459-516.

3. Voutilainen S, Nurmi T, Mursu J, et al. (2006) Carotenoids and cardiovascular health. Am J Clin Nutr 83, 1265-1271.

4. Institute of Medicine, National Academy of Sciences; Food and Nutrition Board, Panel on Dietary Antioxidants and Related Compounds (2000) Dietary Reference Intakes for Vitamin C, Vitamin E, Selenium, and Carotenoids. Washington, DC: National Academy Press.

5. Peng YM, Peng YS, Lin Y, et al. (1995) Concentrations and plasma-tissue-diet relationships of carotenoids, retinoids, and tocopherols in humans. Nutr Cancer 23, 233-246.

6. Natarajan L, Flatt SW, Sun X, et al. (2006) Validity and systematic error in measuring carotenoid consumption with dietary self-report instruments. Am J Epidemiol $\mathbf{1 6 3}$, 770-778.

7. Parker RS (1993) Analysis of carotenoids in human plasma and tissues. Methods Enzymol 214, 86-93.

8. Karppi J, Nurmi T, Olmedilla-Alonso B, et al. (2008) Simultaneous measurement of retinol, $\alpha$-tocopherol and six carotenoids in human plasma by using an isocratic reversedphase HPLC method. J Chromatogr B Analyt Technol Biomed Life Sci 867, 226-232.

9. Mayne ST, Cartmel B, Scarmo S, et al. (2010) Noninvasive assessment of dermal carotenoids as a biomarker of fruit and vegetable intake. Am J Clin Nutr 92, 794-800.

10. Ermakov IV \& Gellermann W (2010) Validation model for Raman based skin carotenoid detection. Arch Biochem Biophys 504, 40-49.

11. Hata TR, Scholz TA, Ermakov IV, et al. (2000) Non-invasive Raman spectroscopic detection of carotenoids in human skin. J Invest Dermatol 115, 441-448.

12. Ermakov IV, Sharifzadeh M, Ermakova M, et al. (2005) Resonance Raman detection of carotenoid antioxidants in living human tissue. J Biomed Opt 10, 064028.

13. Darvin ME, Fluhr JW, Caspers P, et al. (2009) In vivo distribution of carotenoids in different anatomical locations of human skin: comparative assessment with two different Raman spectroscopy methods. Exp Dermatol 18, 1060-1063.

14. Haag SF, Taskoparan B, Darvin ME, et al. (2011) Determination of the antioxidative capacity of the skin in vivo using resonance Raman and electron paramagnetic resonance spectroscopy. Exp Dermatol 20, 483-487.

15. Ermakov IV, Ermakova MR, McClane RW, et al. (2001) Resonance Raman detection of carotenoid antioxidants in living human tissues. Opt Lett 26, 1179-1181.

16. Rerksuppaphol S \& Rerksuppaphol L (2006) Effect of fruit and vegetable intake on skin carotenoid detected by noninvasive Raman spectroscopy. J Med Assoc Thai 89, 1206-1212.

17. Meinke MC, Darvin ME, Vollert H, et al. (2010) Bioavailability of natural carotenoids in human skin compared to blood. Eur J Pharm Biopharm 76, 269-274.

18. Blume-Peytavi U, Rolland A, Darvin ME, et al. (2009) Cutaneous lycopene and beta-carotene levels measured by resonance Raman spectroscopy: high reliability and sensitivity to oral lactolycopene deprivation and supplementation. Eur J Pharm Biopharm 73, 187-194.

19. Hesterberg K, Lademann J, Patzelt A, et al. (2009) Raman spectroscopic analysis of the increase of the carotenoid antioxidant concentration in human skin after a 1-week diet with ecological eggs. J Biomed Opt 14, 024039.

20. Jahns L, Whigham L, Johnson L, et al. (2012) Dermal carotenoids as measured by resonance Raman spectroscopy as a biomarker of response to a fruit/vegetable intervention study. Abstract. Experim Biol 26, 131.3.

21. Institute of Medicine, National Academy of Sciences \& Food and Nutrition Board (2010) Dietary Reference Intakes for Calcium and Vitamin D [AC Ross, CL Taylor, AL Yaktine and HB Del Valle, editors]. Washington, DC: National Academy Press.

22. McCullough ML, Weinstein SJ, Freedman DM, et al. (2010) Correlates of circulating 25-hydroxyvitamin D: cohort consortium vitamin $\mathrm{D}$ pooling project of rarer cancers. $\mathrm{Am}$ $J$ Epidemiol 172, 21-35.

23. Wang Y, Jacobs EJ, McCullough ML, et al. (2009) Comparing methods for accounting for seasonal variability in a biomarker when only a single sample is available: insights from simulations based on serum 25-hydroxyvitamin D. Am J Epidemiol 170, 88-94.

24. Brock K, Huang WY, Fraser DR, et al. (2010) Low vitamin D status is associated with physical inactivity, obesity and low vitamin $\mathrm{D}$ intake in a large US sample of healthy middleaged men and women. I Steroid Biochem Mol Biol 121, $462-466$.

25. Millen AE, Wactawski-Wende J, Pettinger M, et al. (2010) Predictors of serum 25-hydroxyvitamin D concentrations among postmenopausal women: the Women's Health Initiative Calcium plus Vitamin D clinical trial. Am J Clin Nutr 91, 1324-1335.

26. Faure H, Preziosi P, Roussel AM, et al. (2006) Factors influencing blood concentration of retinol, $\alpha$-tocopherol, vitamin $\mathrm{C}$, and beta-carotene in the French participants of the SU.VI.MAX trial. Eur J Clin Nutr 60, 706-717.

27. Galan P, Viteri FE, Bertrais S, et al. (2005) Serum concentrations of beta-carotene, vitamins $\mathrm{C}$ and $\mathrm{E}$, zinc and selenium are influenced by sex, age, diet, smoking status, alcohol consumption and corpulence in a general French adult population. Eur J Clin Nutr 59, 1181-1190.

28. Ermakov IV, Ermakova MR, Gellermann W, et al. (2004) Noninvasive selective detection of lycopene and beta-carotene in human skin using Raman spectroscopy. $J$ Biomed Opt 9, 332-338.

29. Patterson RE, Kristal AR, Tinker LF, et al. (1999) Measurement characteristics of the Women's Health Initiative food frequency questionnaire. Ann Epidemiol 9, 178-187.

30. Schatzkin A, Kipnis V, Carroll RJ, et al. (2003) A comparison of a food frequency questionnaire with a 24-hour recall for use in an epidemiological cohort study: results from the biomarker-based Observing Protein and Energy Nutrition (OPEN) study. Int J Epidemiol 32, 1054-1062.

31. Wang L, Gaziano JM, Norkus EP, et al. (2008) Associations of plasma carotenoids with risk factors and biomarkers related to cardiovascular disease in middle-aged and older women. Am J Clin Nutr 88, 747-754.

32. Locke E, Coronado GD, Thompson B, et al. (2009) Seasonal variation in fruit and vegetable consumption in a rural agricultural community. J Am Diet Assoc 109, 45-51.

33. Ziegler RG, Wilcox HB 3rd, Mason TJ, et al. (1987) Seasonal variation in intake of carotenoids and vegetables and fruits among white men in New Jersey. Am J Clin Nutr 45 , 107-114.

34. Rock CL, Swendseid ME, Jacob RA, et al. (1992) Plasma carotenoid levels in human subjects fed a low carotenoid diet. J Nutr 122, 96-100. 
35. Goralczyk R \& Wertz K (2009) Skin photoprotection by carotenoids. In Carotenoids Volume 5: Nutrition and Health, pp. 335-362 [G Britton, S Liaaen-Jensen and H Pfander, editors]. Basel: Birkhauser Verlag.

36. Someya K, Totsuka Y, Murakoshi M, et al. (1994) The effect of natural carotenoid (palm fruit carotene) intake on skin lipid peroxidation in hairless mice. J Nutr Sci Vitaminol (Tokyo) 40, 303-314.
37. Ribaya-Mercado JD, Garmyn M, Gilchrest BA, et al. (1995) Skin lycopene is destroyed preferentially over beta-carotene during ultraviolet irradiation in humans. J Nutr $\mathbf{1 2 5}$, 1854-1859.

38. Scarmo S, Cartmel B, Lin H, et al. (2010) Significant correlations of dermal total carotenoids and dermal lycopene with their respective plasma levels in healthy adults. Arch Biochem Biophys 504, 34-39. 